\title{
Werkstattberichte
}

\section{Forschendes Lernen im Seminar im Bürgerlichen Recht für Anfänger}

\author{
Reinhard Bork/OlafMuthorst"
}

\section{A. Design}

„Forschendes Lernen“ ist als Konzept fester Bestandteil moderner Hochschuldidaktik, aber weder eine Erfindung aus jüngster Vergangenheit noch im juristischen Studium bereits strukturell verankert. ${ }^{1}$ Der Begriff des forschenden Lernens charakterisiert Lernen durch Merkmale, welche man herkömmlich dem Berufsbild der Forschenden, nicht dem der Studierenden zuordnet. Zu diesen Merkmalen gehören die selbstständige Wahl des Themas, die selbstständige Wahl der Forschungsstrategie mit dem entsprechenden Irrtumsrisiko, aber auch besonderen Chancen unerwarteter Erkenntnisse, die Umsetzung mit wissenschaftlichem Anspruch, die selbstkritische Prüfung des Ergebnisses und eine Präsentation des Erreichten, die eine kritische Einordnung und eine methodische Überprüfung möglich macht. ${ }^{2}$ Von forschendem Lernen spricht man deshalb dann, wenn „die Lernenden den Prozess eines Forschungsvorhabens (...) in seinen wesentlichen Phasen - von der Entwicklung der Fragen und Hypothesen über die Wahl und Ausführung der Methoden bis zur Prüfung und Darstellung der Ergebnisse in selbstständiger Arbeit (...) - (mit)gestalten, erfahren und reflektieren." 3

Reinhard Bork lädt seit mehr als zwei Jahrzehnten im Anschluss an seine im Wintersemester gehaltene Vorlesung „Bürgerliches Recht - Allgemeiner Teil“ besonders begabte Studierende zu einem „Seminar im Bürgerlichen Recht für Anfänger“ für das darauf folgende Sommersemester ein. Ausgewählt werden Studierende, die von den Arbeitsgemeinschaftsleiter/innen empfohlen werden, deren Probe- und/oder Abschlussklausur besonders gut bewertet worden sind oder die in der Vorlesung durch besonders kluge Beiträge aufgefallen sind.

Im Sommersemester 2012 haben die Verfasser dieses Seminar gemeinsam angeboten und es konzeptionell ausdrücklich dem forschenden Lernen gewidmet. Es war schon immer die Idee dieses Seminars, besonders begabte Studierende frühzeitig an die Wissenschaft heranzuführen, indem sie die Gelegenheit erhielten, sich in einem über-

* Prof. Dr. Reinhard Bork ist Inhaber des Lehrstuhls für Bürgerliches Recht, Handels- und Wirtschaftsrecht und Zivilprozessrecht an der Universität Hamburg, Jun.-Prof. Dr. Olaf Muthorst hält eine Juniorprofessur für Bürgerliches Recht mit Zivilprozessrecht ebenda.

1 Vgl. die Beiträge im von Brockmann/Dietrich/Pilniok herausgegebenen Band „Methoden des Lernens in der Rechtswissenschaft“, der die Tagung „Auf dem Weg zur rechtswissenschaftlichen Fachdidaktik: Methoden des Lernens in der Rechtswissenschaft" dokumentiert, die am 29./30. März 2011 in Hamburg stattgefunden hat.

2 Dazu Bundesassistentenkonferenz (BAK), S. 16.

3 Huber, in: ders./Hellmer/Schneider (Hrsg.), S. 9 (11). 
schaubaren Rahmen mit wissenschaftlichen Fragestellungen zu befassen und selbstständig erarbeitete Ergebnisse in einem Seminarreferat zu präsentieren. Bislang wurden den Teilnehmer/innen aber stets die Themen vorgegeben, und im Vordergrund stand die Produktion eines wissenschaftlichen Seminarvortrags. Indem nunmehr das Forschen selbst expliziter Gegenstand der Veranstaltung wurde, wollten die Verfasser beobachten, ob sich dieses geänderte Design auf den Verlauf und die Ergebnisse des Anfängerseminars auswirken würde:

Nach der Auswahl der Studierenden durch die Dozenten wurden die Teilnehmer/ innen in einer ersten Arbeitssitzung nicht mit konkreten Themen, sondern mit dem Prinzip des forschenden Lernens konfrontiert. Im Mittelpunkt stand dabei die Frage, wie man ein noch nicht erschöpfend bearbeitetes, forschungswürdiges Thema findet. Mit dem Auftrag, sich ein solches Thema zu suchen und dazu möglicherweise bereits Arbeitsgruppen zu bilden, wurden die Teilnehmer/innen entlassen. In der zweiten Arbeitssitzung präsentierten die Studierenden einen bunten Strauß interessanter Themen, aus denen dann in einem gemeinsamen Diskussionsprozess die Seminarthemen ausgewählt und Arbeitsgruppen von bis zu drei Studierenden gebildet wurden. Die Arbeitsgruppen hatten mehrere Monate Zeit, sich mit ihren Themen näher zu beschäftigen. In dieser Zeit haben die meisten mindestens einmal Kontakt zu den Dozenten gesucht, um die Ausrichtung des Themas, Gliederung und Aufbau oder sogar einzelne Detailfragen zu besprechen. Am Ende des Projektes stand ein dreitägiges Blockseminar, in dem die Referate im Plenum vorgetragen und diskutiert wurden. Alle Referate wurden auf sehr respektablem Niveau gehalten. Ein Referat ragte besonders heraus, weshalb die Arbeitsgruppe von den Dozenten ermutigt wurde, unter weiterer Betreuung von Olaf Muthorst eine schriftliche Fassung zu erstellen, die schließlich als Beispiel einer besonders gelungenen studentischen Forschungsarbeit in der Fakultätszeitschrift „Hamburger Rechtsnotizen“ veröffentlicht und von den Verfasserinnen auf einem von der Redaktion veranstalteten Symposium vorgestellt wurde.

\section{B. Details}

Im Einzelnen verlief das Projekt wie folgt:

\section{Rekrutierung der Teilnehmer}

Die Dozenten hatten sich von vornherein darauf verständigt, besonders begabte Studierende aus dem zu Ende gehenden 1. Semester auszuwählen. Das geschah weniger, um den Aufwand zu vermeiden, der mit der Auswahl aus einer Vielzahl von Bewerbungen verbunden gewesen wäre, als vielmehr im Interesse der Qualitätssicherung und der Motivation besonders Begabter zu wissenschaftlich anspruchsvoller Arbeit. $\mathrm{Zu}$ diesem Zweck wurden die Leiter/innen der Arbeitsgemeinschaften zur Vorlesung „Bürgerliches Recht - Allgemeiner Teil“ im Wintersemester 2011/2012 schon vor Semesterbeginn gebeten, mit deren Einverständnis solche Studierende zur Teilnahme vorzuschlagen, die in der Arbeitsgemeinschaft durch besondere Leistungen aufgefal- 
len waren. Gleichzeitig wurden diejenigen in die engere Wahl gezogen, die die Probeklausur (Ende Dezember 2011) und/oder die Abschlussklausur (Zwischenprüfungsleistung Mitte Februar 2012) mit mindestens 10 Punkten bestanden hatten. Hinzu kamen einige wenige Personen, die in der Vorlesung durch besondere Leistungen aufgefallen waren. Da der organisatorische Rahmen des am Ende zu besetzenden Blockseminars nicht mehr als sieben Themen zuließ, wurde aus dieser Gesamtgruppe Ende März 2012 ein Kreis von 21 Studierenden zur ersten Sitzung eingeladen.

\section{Erste Arbeitssitzung}

Die erste Arbeitssitzung, an der alle Eingeladenen teilnahmen, fand Anfang April 2012 statt. Die Dozenten machten die Studierenden zunächst mit dem Konzept des Projekts vertraut. Die Idee, ein wissenschaftliches Seminar für Anfänger zu veranstalten und dieses mit dem Konzept des „Forschenden Lernens“ zu verbinden, löste zunächst Überraschung („Wir hatten uns eher ein Vertiefungsseminar vorgestellt“) und Verunsicherung aus („Können wir das schon?“), stießt dann aber doch auf allgemeine Zustimmung.

Im Mittelpunkt der Sitzung stand die Methode der Themenfindung. Dazu wurde ein fiktiver Einstieg gewählt („Stellen Sie sich vor, der Schriftleiter der NJW ruft bei Ihnen an und bittet Sie, einen Aufsatz über ein Thema Ihrer Wahl zu schreiben."). Die Studierenden sollten sich sodann spontan zu Gruppen aus je drei Personen zusammenschließen und in einem fünfminütigen Brainstorming gemeinsam darüber nachdenken, wie man ein geeignetes, d. h. noch nicht hinreichend erforschtes, bearbeitungswürdiges Thema finden könnte. Die erarbeiteten Antworten wurden im Plenum gesammelt und optisch auf einem Flipchart festgehalten. Genannt wurde von den Studierenden

- die Durchsicht von Zeitschriften auf diskussionswürdige Gerichtsentscheidungen und Aufsätze

- die Durchsicht von Lehrbüchern und Kommentaren auf dort benannte offene Fragen

- die Heranziehung eigener oder von dritter Seite herangetragener Alltagsprobleme

- die Berücksichtigung aktueller Entwicklungen, etwa von Gesetzesänderungen oder technischen Innovationen

- die Berücksichtigung eigener fachlicher Interessen

- die Suche im Gesetz nach Regelungslücken

- die Bearbeitung offener eigener Fragen.

Zur Vorbereitung der zweiten Arbeitssitzung wurde dann mit der Erwägung, möglicherweise schon erste Arbeitsgruppen bilden zu können, nach speziellen Vorlieben der Studierenden gefragt, also danach, ob man beispielsweise thematisch dem Stellvertretungsrecht eher als Forschungsgebiet zuneige als dem Vertragsschluss. Da aber keine solche Präferenzen artikuliert wurden, wurden die Studierenden mit dem Auftrag entlassen, sich bis zur nächsten Arbeitssitzung mögliche Themen zu suchen, die für das Projekt geeignet sein könnten. 


\section{Zweite Arbeitssitzung}

Die zweite Plenarsitzung fand 14 Tage nach der ersten statt. Sie war der Themenfindung und der Gruppenbildung gewidmet. Die Teilnehmer/innen wurden zunächst aufgefordert, in der Gruppe darüber zu berichten, für welches Thema sie sich vorläufig entschieden haben und wie sie methodisch auf dieses Thema gestoßen sind. Dabei zeigte sich, dass sich einige Studierende bereits nach der ersten Sitzung spontan zu einer Gruppe zusammengeschlossen und gemeinsam nach einem Thema gesucht hatten; andere sind individuell vorgegangen.

Sechs Studierende hatten sich spontan in zwei Gruppen auf die Suche nach einem Thema gemacht. Bei der einen Gruppe war das Ergebnis ein ganz konkretes (sehr anspruchsvolles) Thema: „Die Sittenwidrigkeit im Wandel der Zeit“. Die Gruppe hatte dieses Thema im Team gesucht, bereits angedacht und erste konkrete Fragestellungen erarbeitet. Die zweite Gruppe hingegen hatte sich noch nicht von Anfang an auf ein Thema geeinigt. Man hatte vielmehr einzeln gesucht, die Ergebnisse in der Gruppe beraten und dann mehrere Themen konsentiert. Interessanterweise wurde das Thema, das die Gruppe schließlich bearbeitete, von den Gruppenmitgliedern zunächst als viel zu schwierig verworfen; erst die Diskussion in der Plenarsitzung und die Ermutigung durch die Dozenten brachte die Gruppe dazu, sich für dieses Thema zu entscheiden.

Die Themensuche gestaltete sich - und zwar ganz unabhängig davon, ob man einzeln oder in der Gruppe tätig wurde - sehr unterschiedlich. Drei Studierende haben spontan zu einem Buch „22 Probleme aus dem BGB, allgemeiner Teil“4 gegriffen, dabei aber den Eindruck gewonnen, dass dort die Streitstände umfassend aufgearbeitet und die Lösungen ganz klar seien. Dieser Weg ist daher von keinem der drei Studierenden weiter verfolgt worden.

Zwei Studierende haben die Inhaltsverzeichnisse der NJW durchgesehen oder eine entsprechende Recherche bei Beck-Online durchgeführt, sind dabei aber zu dem Ergebnis gekommen, dass die Entscheidungen und Aufsätze als viel zu speziell empfunden wurden, so dass dieser Weg nicht weiter verfolgt wurde.

Dreimal wurde als Einstieg die Suchmaschine von Google verwendet. In allen drei Fällen sind dabei Streitstände aufgespürt worden, denen man durch Lektüre der Lehrbücher weiter nachgegangen ist. Drei weitere Studierende haben zunächst die Vorlesungsunterlagen des ersten Semesters durchgesehen und sind von da aus über die Lehrbücher zu konkreten Themen gekommen. In sechs Fällen wurde aus der eigenen Lebenserfahrung oder aus dem Gespräch mit Dritten das Thema geschöpft. Die Studierenden sind hier mit konkreten Rechtsfragen konfrontiert worden, die sie interessant genug fanden, um ihnen nachzugehen. In den allermeisten Fällen haben die Studierenden gleich zu den einschlägigen Lehrbüchern gegriffen und sind dabei auf Themengebiete oder sogar konkrete Themen gestoßen, die sie zur Bearbeitung vorschlagen konnten (neun Antworten).

4 Werner, 22 Probleme aus dem BGB, Allgemeiner Teil. 
In der Summe wird man sagen können, dass in den allermeisten Fällen die Lehrbuchlektüre zum konkreten Themenvorschlag geführt hat. Nur einmal war eine Internetrecherche ausschlaggebend, viermal beruhte die Themenwahl auf eigenem oder fremdem Erleben. Weiterführende Literatur, etwa Monografien oder Aufsätze, haben in diesem Stadium des Prozesses noch keine Rolle gespielt.

Eine erste Sammlung der vorgeschlagenen Themen ergab inhaltlich folgendes Spektrum:

- Internetauktion (Sittenwidrigkeit, Stellvertretung)

- Schadensersatz bei Sportverletzungen

- Internetaktivitäten von Minderjährigen

- Vertragsfreiheit und AGG

- Sittenwidrigkeit im Wandel der Zeit

- Anscheinsvollmacht bei eBay

- Das Erklärungsbewusstsein als Bestandteil der Willenserklärung

- Sonderaspekte der Geschäftsunfähigkeit (relative/partielle Geschäftsunfähigkeit, Altersgeschäftsunfähigkeit)

- Das Verbot des Selbstkontrahierens (Interessenkollision und Identität)

- Zugang und Widerruf bei Willenserklärungen

- Die Rechtsfähigkeit des Menschen angesichts vorgeburtlicher Schädigungen

- Missbrauch der Vertretungsmacht

- Die Untervollmacht

- Rechtsbindungswille und Erklärungsbewusstsein

- Der Tatbestand der Willenserklärung

- Aspekte der Sittenwidrigkeit

- Bereitstellen von Software im Internet

- Auslegung nach Treu und Glauben

- Zeitgemäßheit des Minderjährigenrechts.

Die sich daran anschließende Gruppenbildung und Themenzuweisung bereitete keine besonderen Schwierigkeiten. Zwei Gruppen hatten sich schon im Vorfeld gebildet und konnten sich, ohne dass dies auf Widerspruch anderer Teilnehmer/innen stieß, auf ein gemeinsames Thema einigen. Zwei weitere Gruppen bildeten sich spontan auf persönlicher Ebene und verständigten sich nach kurzer Beratungszeit auf ein Thema. Die beiden verbleibenden Gruppen fanden sich über Themenvorschläge aus dem Kreis der Studierenden zusammen.

Zum Ende der Sitzung wurde das weitere Vorgehen erörtert. Es wurden Möglichkeiten besprochen, wie man nunmehr in die Bearbeitung des Themas einsteigt (Recherchemöglichkeiten, Konkretisierung des Themas, erste Fragestellungen, vorläufige Gliederung, vorläufiger Arbeitsplan, Aufteilung innerhalb der Gruppe). Abschließend wurde den Gruppen freigestellt, nur einen mündlichen Vortrag vorzubereiten oder auch eine schriftliche Fassung des Referats zu erarbeiten. Zudem wurde allen Gruppen angeboten (und nahegelegt), nach der ersten Einarbeitung Kontakt zu 
den Dozenten aufzunehmen und sich bei der weiteren Erarbeitung der Referate beraten zu lassen.

\section{Das Blockseminar}

Das Blockseminar fand auf Bitten der Studierenden und wegen organisatorischer Probleme auf Seiten der Fakultätsverwaltung erst Anfang November 2012 statt. Die Studierenden hatten also hinreichend Zeit, die vereinbarten Themen gründlich zu bearbeiten und den Seminarvortrag angemessen vorzubereiten. Dabei wurde allerdings das Angebot der Dozenten zur Betreuung nur sehr zurückhaltend angenommen. Mehr als die einmalige Besprechung der Themenausrichtung und des Aufbaus anhand einer vorläufigen Gliederung haben die meisten Arbeitsgruppen nicht abgerufen. Nur im Ausnahmefall wurden einzelne Detailfragen im Gespräch mit den Dozenten thematisiert. Unbeschadet dessen waren die Seminarreferate von ausgezeichneter Qualität. Ohne Abstriche kann man sagen, dass das hohe Maß an Selbstständigkeit, das die Studierenden sich abverlangt haben, mit den teilweise noch ungenutzten Optimierungsmöglichkeiten, soweit sie im Folgenden skizziert werden, nicht zu teuer erkauft ist. In aller Kürze lässt sich zusammenfassend Folgendes festhalten:

- Die Sittenwidrigkeit im Wandel der Zeit: Die Referenten hatten sich bewusst ein komplexes und weitgehend unerforschtes Thema gesucht, um erheblichen Konkretisierungsspielraum zu haben. Die Konkretisierung erwies sich aber dann als schwierig und aufwändig; das machte eine Rücksprache mit den Dozenten notwendig. Als problematisch empfand die Gruppe auch die terminliche Koordinierung der Gruppenarbeit, so dass man sich auf die Zuweisung von Teilaspekten einigte. Die Ergebnisse wurden auf hohem Niveau gut verständlich und anschaulich präsentiert. Die Studierenden sind dabei erstaunlich tief eingestiegen und haben ein hohes Maß an Verständnis an den Tag gelegt. Mit dem Begriff des „Wandels" wurde eine unkonventionelle Analysekategorie gewählt und typologisch differenziert.

- Vertragliche Schuldverhältnisse im Vergleich zu Gefälligkeitsverhältnissen: Hier ist das ursprünglich angedachte Thema „Erklärungsbewusstsein und Geschäftswille bei der Willenserklärung“, das sich als zu umfangreich erwies, zu einem dogmatischen Abgrenzungsproblem konkretisiert worden. Dabei drängte sich der Aspekt des Gefälligkeitsverhältnisses in den Vordergrund. Die Gruppe versuchte, aus Einzelfallentscheidungen ein System herauszuschälen und dabei einen neuen Begriff (das „Rechtsfolgenbewusstsein“) einzuführen. Die Begriffsbildung blieb etwas konfus und der Zugriff auf das Thema war methodisch nicht recht konsistent; auch hätte der Meinungsstand umfassender berücksichtigt werden können.

- Der Grundsatz von Treu und Glauben - $\$ 242$ BGB zwischen Einzelfallgerechtigkeit und Rechtssicherheit: Auch hier hatte die Arbeitsgruppe das Thema gegenüber dem ursprünglichen Vorhaben („Auslegung nach Treu und Glauben“) weiter gefasst, es aber bereits auf besondere Analysekategorien konkretisiert. Die Arbeitsgruppe hatte sich darauf verständigt, dass jedes Mitglied zunächst Material und Ideen sammelt. Bei einem Gruppentreffen wurde dann nach „Streitpunkten“ 
gesucht. Durch Lektüre eines Lehrbuchs stieß man auf die „Fallgruppen-Problematik“, bei deren Bearbeitung den Gruppenmitgliedern einzelne Teilaspekte zur Bearbeitung zugewiesen wurden. Die Bearbeitung wies ein sehr hohes methodisches und analytisches Niveau sowie ein hohes Maß an Komplexitätsreduktion auf. Mit der Fallgruppenmethode wurde ein interessanter (Meta-)Aspekt in den Vordergrund der Betrachtung gestellt. Die Detailarbeit wurde durch die Analyse einzelner Entscheidungen geleistet.

- Rechtsscheinhaftung bei unberechtigter Nutzung eines fremden eBay-Kontos: Die Arbeitsgruppe zu diesem Thema berichtete von großen Problemen bei der zeitlichen Koordinierung der Gruppenarbeit, fand dann aber doch zu einer gemeinsamen Arbeitsweise. Man hatte sich vorgenommen, das Thema in der Bearbeitung nicht aufzuteilen, so dass jedes Gruppenmitglied das gesamte Referat hätte allein halten können. Gegenüber der ursprünglichen Fassung des Themas „Die Anscheinsvollmacht bei der Bestellung im Internet" fand sich jetzt eine deutlich breitere rechtliche Einordnung eines konkreteren Problembereichs. Gleichwohl orientierte sich die Gruppe an der Entscheidung BGHZ 189, 346 und nahm sich zum Ziel, den Teilnehmer/innen dieses Urteil verständlich zu machen. Die Materie war gut aufgearbeitet. Die an und für sich hinreichend differenzierte Argumentation hätte allerdings ausgebaut werden können (Schwerpunktsetzung). Das Referat überzeugte durch eine kritische Herangehensweise, ließ allerdings eine wertende Schlussbetrachtung vermissen.

- AGG-Das allgemeine Gleichbehandlungsgesetz: Gegenüber dem ursprünglichen (mehr theoretisch interessierten) Thema („Grundfragen des AGG“) wurden von der Gruppe die Struktur des den Studierenden bisher unbekannten Gesetzes und seine Anwendung im allgemeinen Zivilrecht anhand des „Diskothekenfalls“ (Verweigerung des Zugangs für einen dunkelhäutigen Diskothekenbesucher) in den Vordergrund gestellt (und anhand einer kurzen szenischen Darstellung veranschaulicht). Das bestach durch eine besonders transparente Darstellung, die Veranschaulichung komplexer Normzusammenhänge, ein interaktives Präsentationskonzept und eine sehr differenzierte, kritische und zugleich ausgewogene Analyse des Rechtsstandes. Insgesamt präsentierte diese Arbeitsgruppe, die die gesamte Arbeit im Team geleistet hatte, so dass alle Gruppenmitglieder das Referat allein hätten halten können, die beste Leistung. Sie wurde ermutigt, eine schriftliche Fassung ${ }^{5}$ zu erstellen.

- Minderjährige im Internet: Die Gruppe hat ihr relativ weites Thema frühzeitig konkretisiert und sich entschlossen, sich auf streitige Themen zu konzentrieren, in der Annahme, dass dies das Publikum am ehesten interessieren würde. In mehreren Gruppentreffen wurde die Recherche auf mehrere Schultern verteilt, was zu einer längeren Einzelarbeit ohne weitere Treffen führte. Das gesammelte Material wurde dann aber in der Gruppe ausgewertet und das Referat hätte auch hier jedes Gruppenmitglied allein halten können. Das Ergebnis überzeugte durch eine ausgesprochen eigenständige Annäherung an das Thema und einen innovativen Lö- 
sungsansatz zu bislang unentschiedenen Fallgruppen. Zwar wurde das Differenzierungspotential nicht unbedingt ausgeschöpft. Insgesamt wurden aber die Probleme in den Kontext eingeordnet und kritisch beurteilt.

\section{Auswertung}

In der Gesamtschau kann das Projekt nur als gelungen bezeichnet werden. Auswahlsystem und Veranstaltungsformat (Blockseminar) haben sich in diesem Zusammenhang bewährt. Die Studierenden haben sich, anders als in einem „normalen“ Seminar, nicht nur vertieft mit einem rechtswissenschaftlichen Thema, sondern auch mit rechtswissenschaftlicher Forschung als Reflexionsgegenstand beschäftigt. Freilich zeigte sich in der Abschlussdiskussion, dass die Erwartungen der Studierenden anfangs zu hoch gesteckt waren. Sie waren davon ausgegangen, dass von ihnen erwartet werde, etwas „Neues“ zu finden. Sie artikulierten als anfängliches Problem, dass es in der Rechtswissenschaft gar nicht so viel Neues gebe, und die ursprüngliche Vorstellung, dass das Neue nicht im Referat, sondern erst in der Diskussion herausgearbeitet werden könne. Am Ende hatten die Studierenden sehr viel konkretere Vorstellungen davon, was Forschung eigentlich sein könnte. Die Gruppe einigte sich auf drei wichtige Aspekte wissenschaftlichen Arbeitens (Themensuche, kritische Beleuchtung der vorhandenen Quellen, Ansatz zu neuen Lösungen) und war der Auffassung, dass auch die verständliche Aufbereitung des status quo, also eines Meinungsstandes, Ergebnis rechtswissenschaftlicher Forschung sein könne. Die Teilnehmer sahen jetzt vieles kritischer, und es konnte konstatiert werden, dass das Interesse an der Wissenschaft geweckt oder, sofern es schon bestand, gestärkt war.

In einem reflektierteren Verständnis von wissenschaftlichem Arbeiten und einer erhöhten Identifikation mit der eigenen wissenschaftlichen Arbeit dürfte ein Ertrag liegen, der bei Anfängerseminaren früherer Semester nicht in gleicher Sichtbarkeit erzielt wurde, weil dort die Methode des Arbeitens oft nur indirekt zum Thema gemacht wurde und die Seminarleistung als Ergebnis im Mittelpunkt stand, während die eigentliche Forschungsarbeit gleichsam außerhalb des Seminars geleistet wurde. Im Vergleich zu den Anfängerseminaren früherer Semester, die nicht ausdrücklich als forschendes Lernen angelegt waren, kann man aber umgekehrt festhalten, dass die von den Studierenden gewählten Themen ebenso geeignet gewesen sind wie die Themen, die in früheren Semestern von Seiten der Dozenten zur Bearbeitung vorgegeben worden sind. Die selbst gewählten Themen lagen auch inhaltlich sehr dicht bei den Themen, die sonst für Anfängerseminare gestellt worden sind. Diese Themen waren sehr weit gesteckt. Entsprechend sind auch eher generelle Ergebnisse erarbeitet worden. Innovativer wären die Arbeiten als Forschungsprojekte aufs Ganze gesehen vermutlich dann verlaufen, wenn mehr im Detail gearbeitet worden wäre. Das Niveau der erzielten Ergebnisse und ihrer Präsentation entsprach aber wiederum sehr gut dem, was man sonst in Anfängerseminaren der traditionellen Art erwarten kann. Betrachtet man, dass diese Ergebnisse in hohem Maße selbstständig gewonnen wurden, ist das keine geringe Leistung. 


\section{Literaturverzeichnis}

Brockmann, Judith/Dietrich, Jan-Hendrik/Pilniok, Arne (Hrsg.), Methoden des Lernens in der Rechtswissenschaft, Baden-Baden 2012.

Bundesassistentenkonferenz (BAK), Forschendes Lernen - Wissenschaftliches Prüfen, Neuauflage, Bielefeld 2009.

Haller, Annika/Hüttmann, Malin/Linn, Merle, Das Allgemeine Gleichbehandlungsgesetz - Ein Überblick über die zivilrechtlichen Regelungen, in: Hamburger Rechtsnotizen (HRN) 2013, S. 19-26.

Huber, Ludwig, Warum Forschendes Lernen nötig und möglich ist, in: ders./Hellmer/Schneider (Hrsg.), Forschendes Lernen im Studium: Aktuelle Konzepte und Erfahrungen, Bielefeld 2009, S. 9-35.

Werner, Olaf/Neureither, Georg, 22 Probleme aus dem BGB, Allgemeiner Teil, 7. Aufl., Neuwied 2005. 